\title{
Anisotropy of some FCC transition metals.
}

\author{
Fae’q AA Radwan*
}

Faculty of Engineering, Near East University, Turkey

\begin{abstract}
The norm of elastic constant tensor and the norms of the irreducible parts of the elastic constants of FCC elemental transition metals Nickel, Copper, Silver, Gold, Platinum and Rhodium are calculated. The relation of the scalar parts norm and the other parts norms and the anisotropy of these elements are presented. The norm ratios are used to study anisotropy of these elements.
\end{abstract}

Keywords: Transition elements isotropy, Norm, Anisotropy, and Elastic constants.

Accepted on October 04, 2017

\section{Introduction}

Anisotropy and Isotropy: when the properties of a material vary with different crystallographic orientations, the material is said to be anisotropic, common examples of anisotropic materials are wood and composites. Alternately, when the properties of a material are the same in all directions, the material is said to be isotropic.

The decomposition procedure and the decomposition of elastic constant tensor is given by Radwan [1], also the definition of norm concept and the norm ratios and the relationship between the anisotropy and the norm ratios are given by Radwan [1]. As the ratio becomes close to one the material becomes more isotropic, and as the ratio becomes close to one the material becomes more anisotropic as explained by Radwan [1].

\section{Calculations}

By using Table 1 and the decomposition of the elastic constant tensor, we have calculated the norms and the norm ratios as is shown in Table 2 .

Table 1. Elastic constants (GPa) [2].

\begin{tabular}{|c|c|c|c|c|}
\hline No, & FCC, Transition Metal & $\boldsymbol{C}_{\mathbf{1 1}}$ & $\boldsymbol{C}_{\mathbf{1 2}}$ & $\boldsymbol{C}_{\mathbf{4 4}}$ \\
\hline $\mathbf{1}$ & $\mathbf{N i}$ & 230.69 & 177.82 & 80.10 \\
\hline $\mathbf{2}$ & $\mathbf{C u}$ & 169.81 & 129.76 & 57.87 \\
\hline $\mathbf{3}$ & $\mathbf{A g}$ & 140.98 & 96.12 & 59.27 \\
\hline $\mathbf{4}$ & $\mathbf{A u}$ & 179.42 & 147.38 & 41.64 \\
\hline $\mathbf{5}$ & $\mathbf{P t}$ & 313.99 & 257.92 & 73.69 \\
\hline $\mathbf{6}$ & $\mathbf{R h}$ & 339.62 & 232.29 & 142.58 \\
\hline
\end{tabular}

Table 2. The norms and norm ratios.

\begin{tabular}{|l|c|c|c|c|c|c|c|c|}
\hline No. & $\begin{array}{c}\text { FCC, Transition } \\
\text { Metal }\end{array}$ & $N_{s}$ & $N_{n}$ & $N_{s}$ & $N$ & $\frac{N_{d}}{N}$ & $\frac{N_{d}}{N}$ & $\frac{N_{n}}{N}$ \\
\hline $\mathbf{1}$ & $\mathbf{N i}$ & 617.738 & 0 & 98.37 & 625.521 & 0.9876 & 0 & 0.1573 \\
\hline $\mathbf{2}$ & $\mathbf{C u}$ & 452.118 & 0 & 69.371 & 457.409 & 0.9884 & 0 & 0.1517 \\
\hline $\mathbf{3}$ & $\mathbf{A g}$ & 364.488 & 0 & 67.529 & 370.691 & 0.9833 & 0 & 0.1822 \\
\hline $\mathbf{4}$ & $\mathbf{A n}$ & 485.476 & 0 & 46.962 & 487.742 & 0.9954 & 0 & 0.0963 \\
\hline $\mathbf{5}$ & $\mathbf{P t}$ & 849.949 & 0 & 83.687 & 854.059 & 0.9952 & 0 & 0.098 \\
\hline $\mathbf{6}$ & $\mathbf{R h}$ & 878.806 & 0 & 162.45 & 893.695 & 0.9833 & 0 & 0.1818 \\
\hline
\end{tabular}

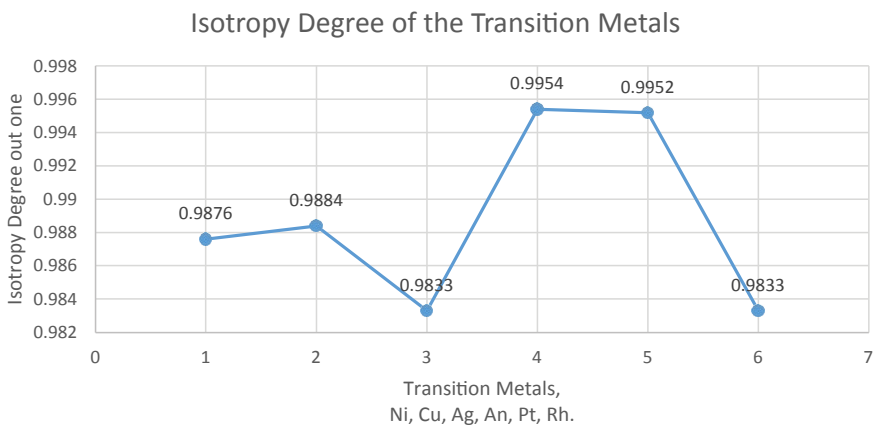

Graph 1. Isotropy degree of the transition metals.

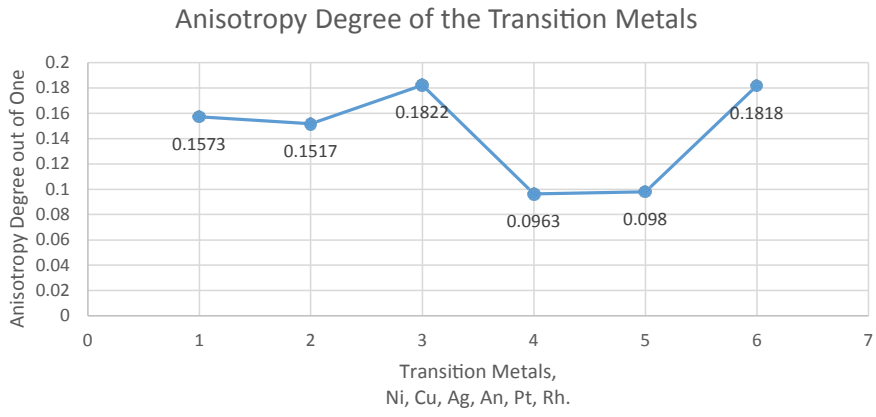

Graph 2. Anisotropy degree of the transition metals.

Elastically Strength of the Transition Metals

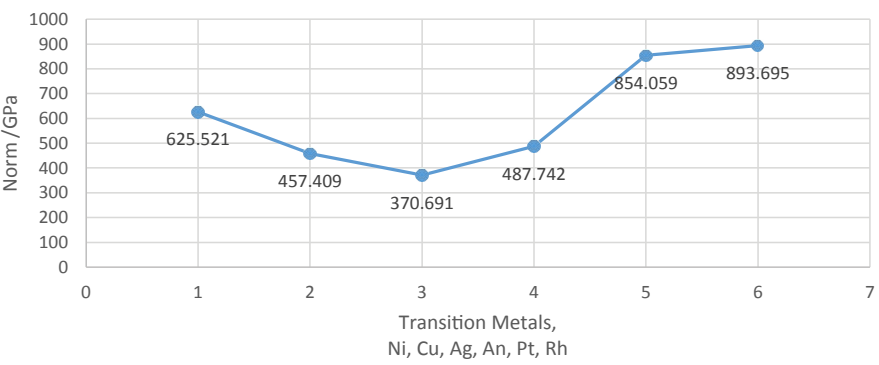

Graph 3. Elastically strength of the transition metals.

\section{Conclusion}

We can conclude from table 2, and the above graphs (Graphs 1-3)by considering the ratio $\frac{N_{s}}{N}$, that Gold An, is the most isotropic element, and the most anisotropic element is Rhodium Rh, because the value of $\frac{N_{n}}{N}$ is the smallest and 
the value of $\frac{N_{n}}{N}$ is the largest for Rhodium $\mathrm{Rh}$.. Also we can notice by considering the value of $N$ that this value is the highest (893.695) in the case of the Rhodium Rh element, so we can say that Rhodium $\mathrm{Rh}$ is elastically the strongest, and the in the case of the element Silver $\mathrm{Ag}(370,671)$ the value of $N$ is the smallest so we can say that Silver Ag, elastically is the least strong element [2].

\section{References}

1. Radwan FAA. Norm ratios and anisotropy degree. J Appl Sci. 2001;1(3):301-4.

2. Sutton AP. Long-range Finnis-Sinclair potentials. Philos Mag Lett. 1990;61:139-46.

\section{*Correspondence to:}

Fae`q AA Radwan

Faculty of Engineering

Near East University

Turkey

faeq.radwan@neu.edu.tr 